\title{
TCAS Solution to Reduce Alarm Rate in Cockpit and Increase Air Safety
}

\author{
Abdelali Achachi ${ }^{1}$ and Djamel Benatia ${ }^{2}$ \\ Department of Electrical Engineering, Batna University, Algeria \\ Idabt@enna.dz,2dj_benatia@yahoo.fr
}

\begin{abstract}
Over the years, air traffic has continued to increase. The development of modern systems has made it possible to cope with this increase, whilst maintaining the necessary level of safety. The Traffic Alert and Collision Avoidance System (TCAS) has been developped to reduce the risk of mid-air collision and is currently mandated on all large transport aircraft This system is based on interrogations to determine the altitude and relative positions of nearby aircraft. Despite technical advances in ATC systems, there are cases when the separation provision fails due to a human or technical error. Any separation provision failures may result in an increased risk of a midair collision. These systems would generate a high rate of unnecessary alarms especially in dense terminal areas and pilots become stressed and wrong actions can be made. A new design of TCAS is investigated in this paper to compensate for any limitations of ATC performance and decrease the rate of unnecessary alerts. It is based on transmitting altitudes and GPS coordinates between aircraft transponders to determine the accurate positions and directions of movement of nearby aircraft.
\end{abstract}

Keywords: GPS coordinates; mode S transponder; Transmitting data; TCAS; Air Traffic Control.

\section{Introduction}

The Traffic Alert and Collision Avoidance System (TCAS) [1] is an aircraft system based on secondary surveillance radar (SSR) [2] transponder signals which operates independently of ground based equipment to provide advices and information to the pilot on potential conflicting aircraft that are equipped with SSR transponders.

The TCAS II is the current system commercially available that corresponds to the requirements for ACAS II. The current version of TCAS II is Version 7. This new version includes significant safety, operational and technical improvements comparing to previous version.

TCAS II interrogates and receives via the aircraft transponder Mode C and Mode S [3] messages. The interrogation rate is about $1 \mathrm{~Hz}$ and the logic tests for a potential threat are based on difference of altitudes and relative position. Once an aircraft is detected by the TCAS and determined to be a potential threat, TCAS presents it to the pilot as a Traffic Advisory (TA). If the threat becomes imminent then TCAS II proposes an avoidance manoeuvre to the pilot in the vertical sense, this is called a Resolution Advisory (RA).

This paper aims to reduce some current TCAS weaknesses such as high rate of unnecessary alerts which disturb pilots and lead them to make unnecessary manoeuvres. The positions of nearby aircraft presented by TCAS are relative and not accurate, they are provided by a directional antenna (four directions), and therefore one aircraft can be found in an angle of 90 degrees, this is shown in Figure 1. Resolution Advisory in the horizontal sense and the direction of movement of other aircraft are not provided by the current TCAS. 


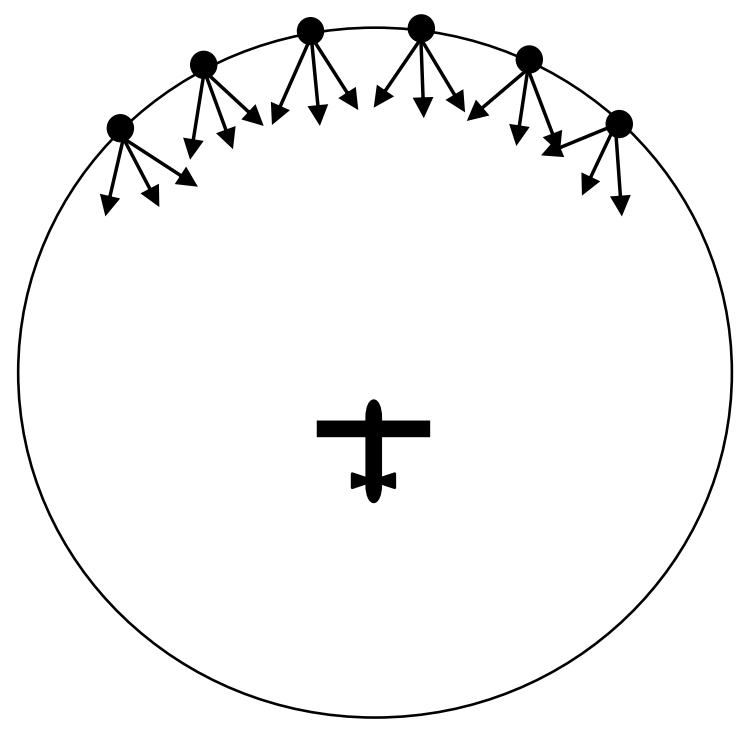

Figure 1. Many Possible Positions and Directions for One Aircraft Shown by Current TCAS

The proposed system uses the ADS-B (Automatic Dependent Surveillance-Broadcast) [45]; it can be used to improve the monitoring of traffic in domestic airspace. It should be noted here that this process is available and should be adopted worldwide for the data link ADS-B.

A global navigation system will help standardize procedures and displays in the cockpit. The ultimate goal is to improve the current systems and eliminate unnecessary alarms. It uses some SSR radar principles, for that some basic data are needed such as altitude (flight level) provided by the barometric systems aboard aircraft; the position of each plane provided by three satellites; the trajectory (direction) provided by two successive positions, and the ground speed calculated with the distance traveled during a definite time. The same technique is used by SSRs and we use this technique to perform our system.

This paper is organized as follows: Section 2 presents the data model and lists the assumptions and some preliminary notions; Section 3 presents the new system function; Section 4 presents the simulations and discussions and the last Section will be the conclusion.

\section{Data Model and Preliminary}

Three levels of ACAS [1] have been described by International Civil Aviation Organization (ICAO): ACAS I, ACAS II and ACAS III.

- ACAS I is an ACAS which provides information as an aid to "see and avoid" action but does not include the capability for generating Resolution Advisories (RAs).

- ACAS II provides vertical resolution advisories (RAs) in addition to traffic advisories (TAs).

- ACAS III is an ACAS which provides vertical and horizontal resolution advisories (RAs) in addition to traffic advisories (TAs).

- ACAS X [6] represents a main revolution in how the advisory logic is generated and represented. It represents the logic using a numeric table that has been optimized with respect to models of the airspace.

\subsection{Technical Description of TCAS II}

TCAS II consists of the Mode S transponder with a Control Panel, the TCAS computer unit which performs airspace surveillance; threat detection; avoidance manoeuvre determination and the generation of advisories [7]. 
The antennas used by TCAS II are a directional antenna that is mounted on the top of the aircraft and either an omni-directional or a directional antenna mounted on the bottom of the airplane.

These antennas transmit interrogations on $1030 \mathrm{MHz}$ at varying power levels in each of four 90 degrees azimuth segments. These antennas also receive transponder replies, at 1090 $\mathrm{MHz}$, and send these replies to the TCAS computer.

Two more antennas are also required for the Mode $S$ transponder. One antenna is mounted on the top of the aircraft and the other is mounted on the bottom. These antennas enable the Mode $\mathrm{S}$ transponder to receive interrogations at $1030 \mathrm{MHz}$ and reply to the received interrogations at $1090 \mathrm{MHz}$.

Connection with the transponder (Mode $S$ ) to coordinate resolution advisories, in case of all aircraft are equipped with TCAS II; connection with the alticoder to obtain pressure altitude; Connection with the radar altimeter in order to prevent wrong RAs when the aircraft is too close to the ground and determine if aircraft tracked by TCAS are on ground.

\subsection{Reply Structure}

A mode $\mathrm{S}$ structure response is $p_{S}(\mathrm{t})$ which has the form of pulses [8] of the mode $\mathrm{S}$. According to the ICAO (International Civil Aviation Organization) requirements; it must meet the following constraints presented on Table 1:

Table 1. Mode S Reply Structure

\begin{tabular}{|l|c|c|c|}
\hline Time $(\mu \mathrm{s})$ & $\mathrm{t} \leq 0$ & $0,1 \leq \mathrm{t} \leq 0,5$ & $\mathrm{t} \geq 0,6$ \\
\hline $\mathrm{p}_{\mathrm{s}}(\mathrm{t})$ & 0 & 1 & 0 \\
\hline
\end{tabular}

All transmitted data are coded according to the following equations. The emission stream of bits consists of a preamble followed by bits of data.

$\tilde{b}=\left[1,0,10,0,0,1,0,1,0,0,0,0,0,0,0, b_{1}, b_{2}, \ldots \ldots ., b_{56 / 112}\right]$

The preamble has to facilitate synchronization (detection of the beginning of a structure). The response signal emitted by the Mode $\mathrm{S}$ transponder is a pulse amplitude modulation and it has the following form:

$b_{S}(t)=\sum_{n=0}^{127 / 239} \tilde{\mathrm{b}}(n) \cdot p_{S}\left(t-\frac{1}{2} n T_{S}\right)$

Where $\tilde{b}(n)$ is the $\mathrm{n}^{\text {th }}$ input and $\mathrm{T}_{\mathrm{S}}=1 \mu \mathrm{s}$ is the period of Mode $\mathrm{S}$.

\section{The New System Function}

This system will certainly allow pilots to participate in new arrangements for collaboration with air traffic controllers, procedures that increase security and limit delays. This technology allows pilots to receive clear visibility in the surrounding air traffic, either in the air or ground. The new instrument combines the technology of air traffic ADS-B [4-5] to that of the TCAS collision avoidance. On board, the Mode $\mathrm{S}$ transponder transmits the following information: position; identification and flight level or altitude, but the course, speed and trajectory are calculated by the system according to the geographical coordinates, allowing the pilot to identify other aircraft equipped with transponders and to know accurately their positions and trajectories. The display allows pilots to bring out on their screens and other equipment to maintain a fixed distance. It also allows pilots to view a moving map extremely precise area of the airport, showing traffic conditions with the aircraft moving on the ground 
or close to it. The goal is to give pilots the best possible information to make safe, fast and accurate decisions.

\subsection{Technical Description of the Proposed System}

The antenna used by this system is the same of the transponder's one. It is an omnidirectional antenna that is mounted on the top and on the bottom of the aircraft. The use of the directional antenna on the bottom of the aircraft is not needed.

The geographical coordinates of nearby aircraft are updated each second; the new and the previous position are used to show the direction of movement of each aircraft on TCAS screen.

The system geometry is shown in Figure 2 (b) and the distance between two points is given by the following formula [9] resulting from the spherical trigonometry:

$$
P=60 \cos ^{-1}\left(\sin L_{A} \sin L_{B}+\cos L_{A} \cos L_{B} \cos g\right)
$$

$P$ is expressed in nautical miles (NM).

The ground speed is given for each aircraft by the following formula:

$G S=\frac{60 \cos ^{-1}\left(\sin L_{A} \sin L_{B}+\cos L_{A} \cos L_{B} \cos g\right)}{\tau}$

The Convergence rate $(\mathrm{Cr})$ is given by the following equation:

$$
\begin{aligned}
& C r=\frac{P_{n}-P_{n+1}}{\tau} \\
& C r=60 \frac{\cos ^{-1}\left(\sin L_{A n} \sin L_{B n}+\cos L_{A n} \cos L_{B n} \cos g_{n}\right)-\cos ^{-1}\left(\sin L_{A n+1} \sin L_{B n+1}+\cos L_{A n+1} \cos L_{B n+1} \cos g_{n+1}\right)}{\tau}(6)
\end{aligned}
$$

$\mathrm{Cr}$ must be taken into consideration because it represents the approaching speed of one aircraft towards the others; it means that aircraft are face to face when it is high. Where $\tau$ represents the time between two successive points.

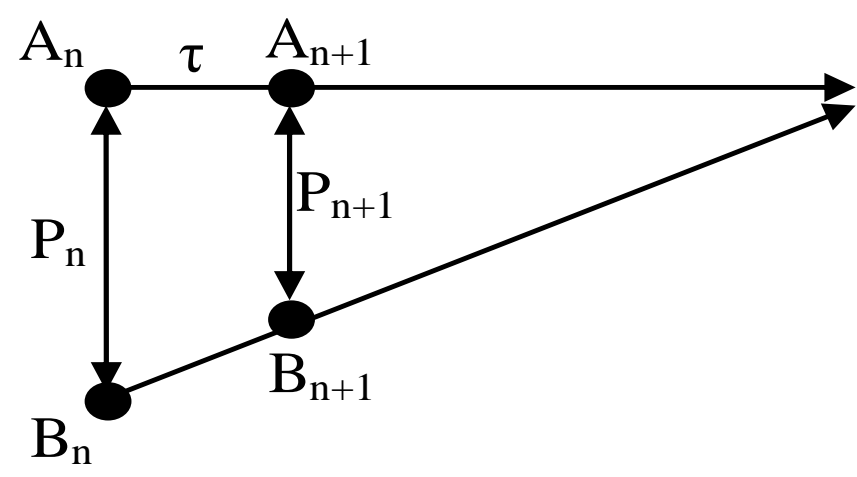

(a) 


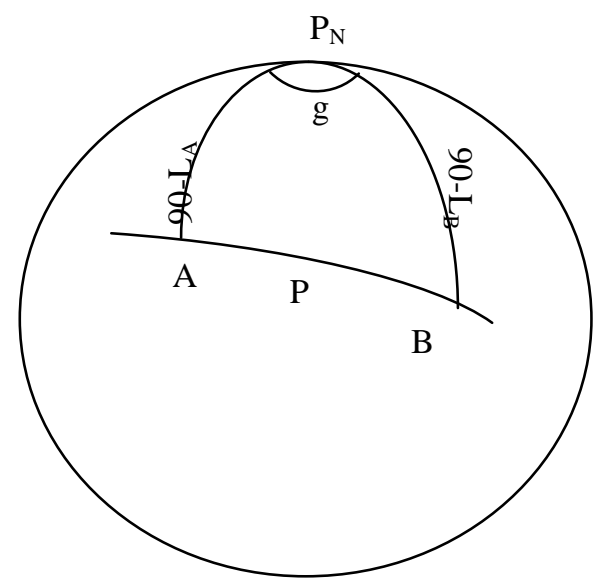

(b)

Figure 2. Distance and Earth Geometry

The goal is to determine the altitude, position, true course (TC) which is defined as a track of each aircraft in order to acquire all necessary data by sending interrogations and all aircraft entering this zone are identified by its mode $\mathrm{S}$ addresses. This is achieved by assigning to each aircraft a unique address. This address is composed of 24 bits. Its data are exchanged with all aircraft and also with ground stations.

\subsection{The Collision Avoidance Logic}

The collision avoidance logic [10] or CAS (Collision Avoidance System) logic is based on two basic concepts: the sensitivity level and the warning distance. Although the CAS parameters are strictly defined, the complexity of collision avoidance logic makes prediction of exact behaviour in real time as shown in Figure 3.

The sensitivity level is a function of the altitude and defines the level of protection. The warning distance is mainly based, in our system, on the estimated distance-to-go (and not time-to-go) to the Closest Point of Approach (CPA). The warning time allows for additional range protection in case of low closure rates.

The CAS logic computes the closure rate of each target within surveillance range, in order to estimate the time in seconds to CPA, and the horizontal miss distance at CPA. 


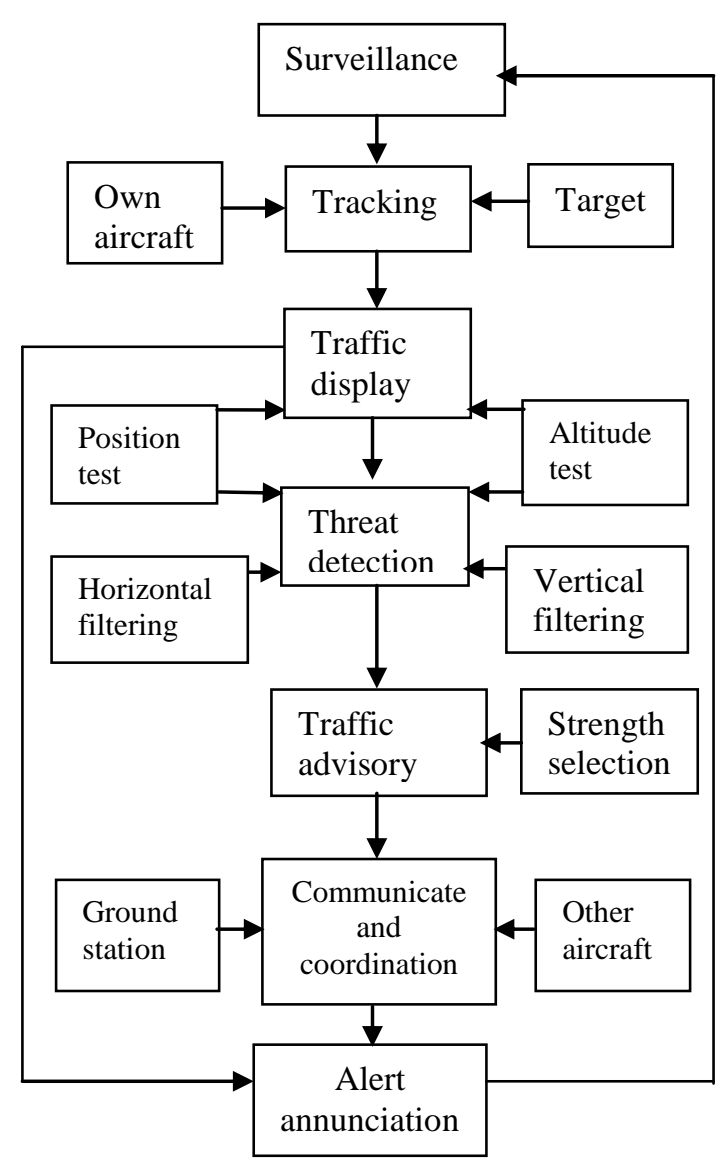

Figure 3. CAS Logic Functions

The required accuracy of location cannot be obtained from a single satellite. Therefore, a multiple coverage is required to locate a plane by triangulation and to ensure multiple visibilities from any point on earth which aims to implement the global navigation satellite. An aircraft must have a permanent visibility with a number of GPS satellites more than or equals to 4.

Each point (latitude and longitude) contains 19 digits coded on 76 bits transmitted in binary code that pulse has a duration of $23.75 \mu \mathrm{s} 76$ bits, it is modulated by differential phase DPSK (Differential Phase Shift Keying). The TCAS unit calculates the distance between it and the other aircraft, so all conflicting aircraft are displayed on a screen (only aircraft at \pm 2000 feet are displayed).

The main problem of the ADS-B technique is this added verbosity of the extra information transmitted, which is considered unnecessary for collision avoidance purposes. The more data transmitted from one aircraft in accordance with the system design, the lesser the number of aircraft that can participate in the whole system, due to the fixed and limited channel data bandwidth (1 megabit/second with the 26/64 data bits to packet length bit capacity of the Mode S downlink data format packet). For every Mode S message of 64 bits, the overhead demands 8 for clock synchronic at the receiver and Mode S packet discovery, 6 for type of Mode S packet, 24 for who it came from. Since that leaves only 26 for information, multiple packets must be used to convey a single message.

This system represents a valid means for transmitting data on the frequencies $1030 \mathrm{MHz}$ and $1090 \mathrm{MHz}$ through very short time, so we will have several interrogations and answers in a second. It represents also the algorithmic foundations of the precise immediate trajectory reconstruction process [11] used to find reference position from transmitted measures. These positions will serve as a reference for the evaluation of the accuracy of CAS logic functions. 
These frequencies (1030 and $1090 \mathrm{MHz}$ ) are busy by interrogations and replies sequence provided by TCAS and several other critical systems including ground based radar, wide area multilateration operate independently but rely on the same RF channels as TCAS. To ensure good operation of these systems, it is important that each system does not prevent transmitting data of the other ones. Thus, it is necessary for each system to reduse its transmissions [12] to the shared communication channel.

\subsection{Resolution Algorithm and Priority}

The following rules [10] take into account the maneuverability and the phase of flight of aircraft involved and the required vertical separation is not respected. We can establish three rules based on horizontal geometry.

- Rule 1: "face to face" both aircraft have no priority, and have to give way and turn right to avoid conflict. This must be applied when the difference of route is between 135 to 180 degrees.

- Rule 2: If two aircraft have " crossing ways", the aircraft coming to see approaching aircraft at right hand side should turn left and the other should turn right to avoid conflict to the crossing. This has to be applied when the difference of route is between 15 to 135 degrees.

- Rule 3: "Overtaking" the aircraft that is behind has to turn 15 degrees right to pass. This will be applied when the difference of route is between 0 to 15 degrees.

\subsection{Conflict Detection}

Assume that the airspace is represented as a three dimensional Cartesian Coordinates System, the position and velocity for the ownership are: (L, G, Alt) and $\left(\mathrm{V}_{\mathrm{ox}}, \mathrm{V}_{\mathrm{oy}}, \mathrm{V}_{\mathrm{oz}}\right)$ and for the intruder are $\left(\mathrm{L}_{\mathrm{i}}, \mathrm{G}_{\mathrm{i}}, \mathrm{Alt}_{\mathrm{i}}\right)$ and $\left(\mathrm{V}_{\mathrm{ix}}, \mathrm{V}_{\mathrm{iy}}, \mathrm{V}_{\mathrm{iz}}\right)$ respectively. These terms are all time variant; the protected area in cylindrical column has $\mathrm{R}$ in radius with $\mathrm{H}$ in high as shown in Figure 4. When the intruder intercepts the protected area of ownership, it means a conflict threat. To avoid mid-air collision, after conflict detection, a resolution is necessary before it happens.

As long as the pilot can see the serrounding aircraft, we assume that the visuel sign starts when the conflicting traffic is at \pm 1200 feet and the audio alarm starts at \pm 950 feet insteed of \pm 1200 feet, this will reduce alarms significantly and avoid unnecessary alarms especially in holding patterns.

In the current system the audio alarm starts at about $3 \mathrm{NM}$. We assume that horizontal conflict is detected as visuel announcement at $R=3 \mathrm{NM}$ and audio alarm starts at $r=2 \mathrm{NM}$ as shown in Figure 4. The reducing space is given by the following equation :

$$
\frac{S_{r}}{S_{R}}=1-\left(\frac{2 R x+R \Delta h+H x-x^{2}-x \Delta h}{R(R+H)}\right)
$$

Where :

$S_{R}:$ represents the original alert airspace

$\mathrm{S}_{\mathrm{r}}$ : represents the reduced alert airspace 


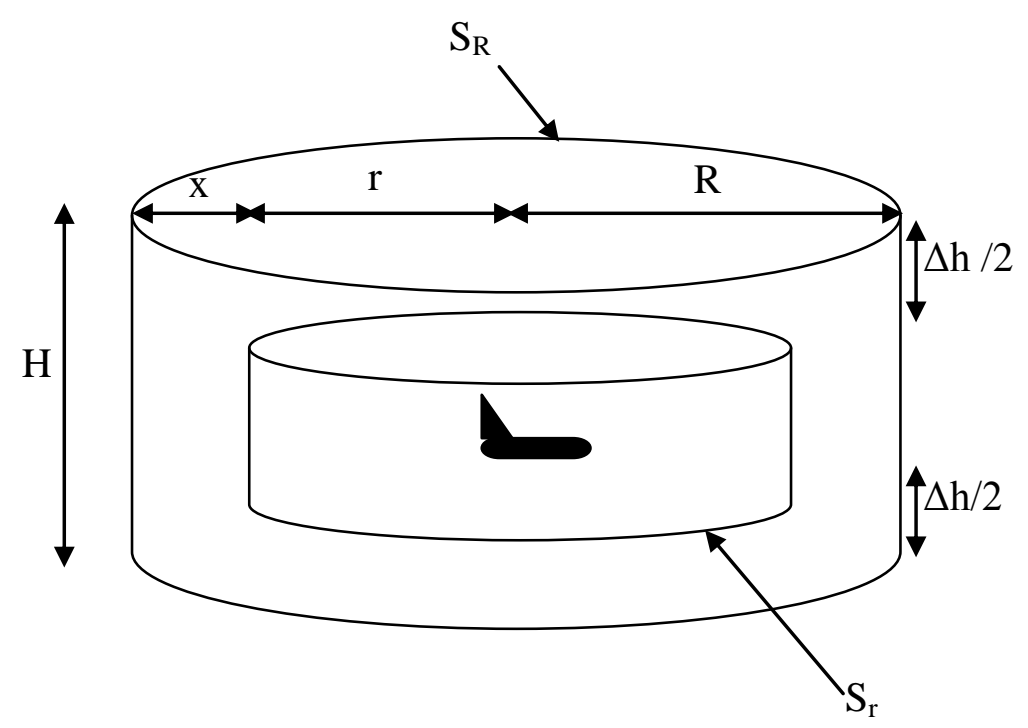

Figure 4. Reducing Vertical and Horizontal Space

When we reduce the audio alarm distance by $x=1 \mathrm{NM}$ and the altitude till \pm 950 feet, the reducing space will be more than $54.5 \%$; this means that the unnecessary audio alarms will be decreased by : $P=0.545$.

\section{Simulations and Discussions}

In the TCAS simulations, METLAB 9 is used to test the proposed TA and RA algorithm using random flight data but they can be encountered in real situation. Consider the conflicting flights in Northeast sector of Algeria, vertical separation is applied in first priority, then horizontal separation is applied in second priority. All simulations are applicable for all aircraft type; at low and high altitude.

A high fidelity TCAS Surveillance Simulation has been developed over the last two decades to analyze the impact of changes to the TCAS surveillance algorithms. These aircraft tracks are typically derived from a ground based radar data source. The environment within the simulation operates cycle, the position of each aircraft is updated according to the input dataset. Interrogation and reply sequences for each TCAS aircraft are then simulated.

Once a threat is detected, the first solution to be taken is vertical resolution to maintain vertical separation between aircraft. If not possible, horizontal resolution must be adopted as illustrated in Figure 5. Aircraft 1 and aircraft 2 are converging to conflict point; the avoiding action must be done following Rule 2 . 


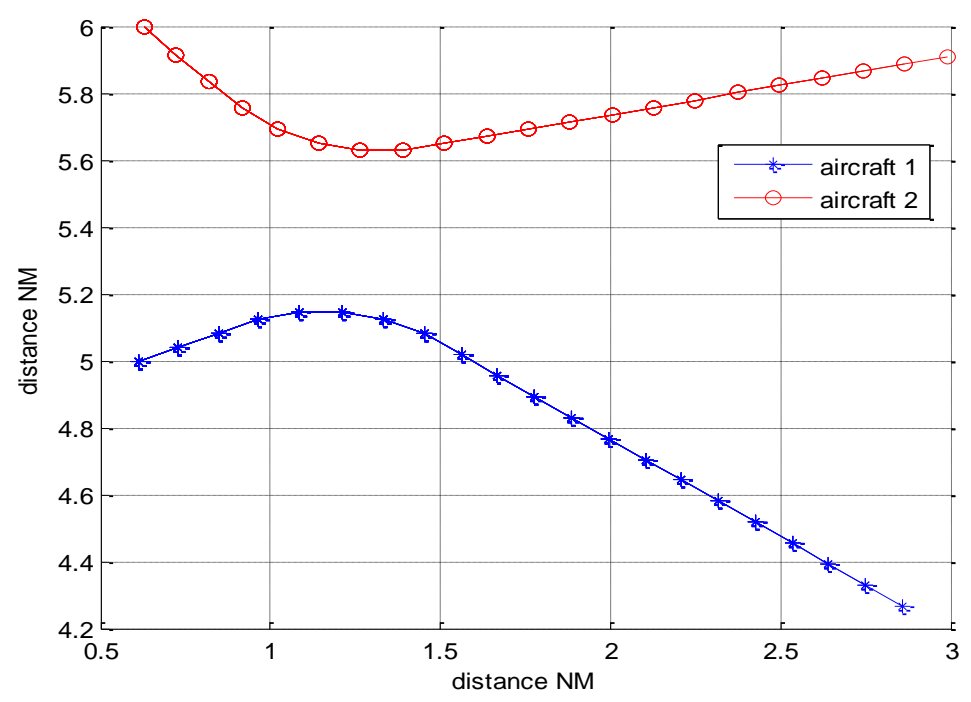

Figure 5. Horizontal Resolution

Some conflicts need both resolutions to solve the problem as shown in next simulations.

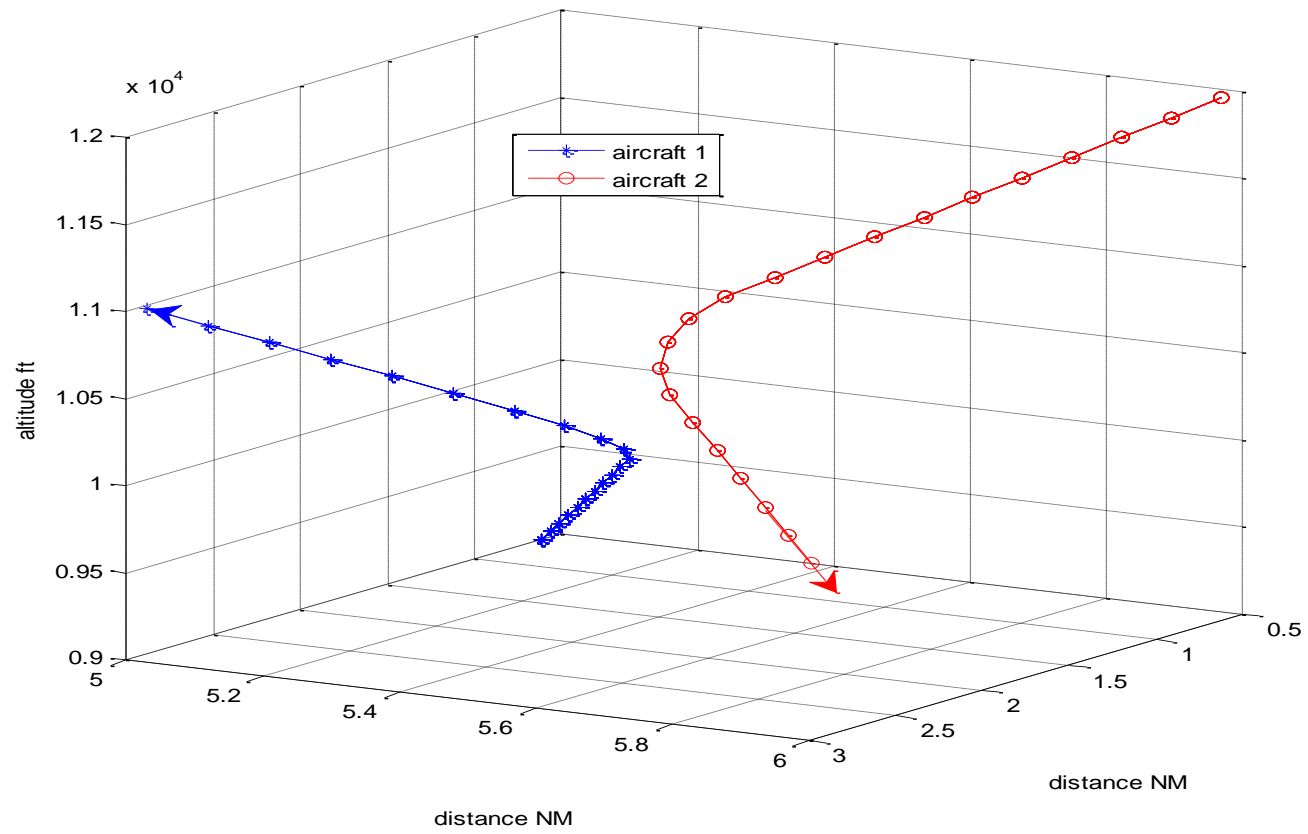

Figure 6. Vertical and Horizontal Resolution

Aircraft 1 was given clearance to climb to FL120 and aircraft 2 was given clearance to descend to FL090. As aircraft 1 and 2 approach the cleared level FL105 at a high vertical rate (and therefore threatening to bust the required separation) TCAS issues an RA to decrease vertical speed and aircraft 1 has to go right and continue climbing, The aircraft 2 has to go left and continue descending. 


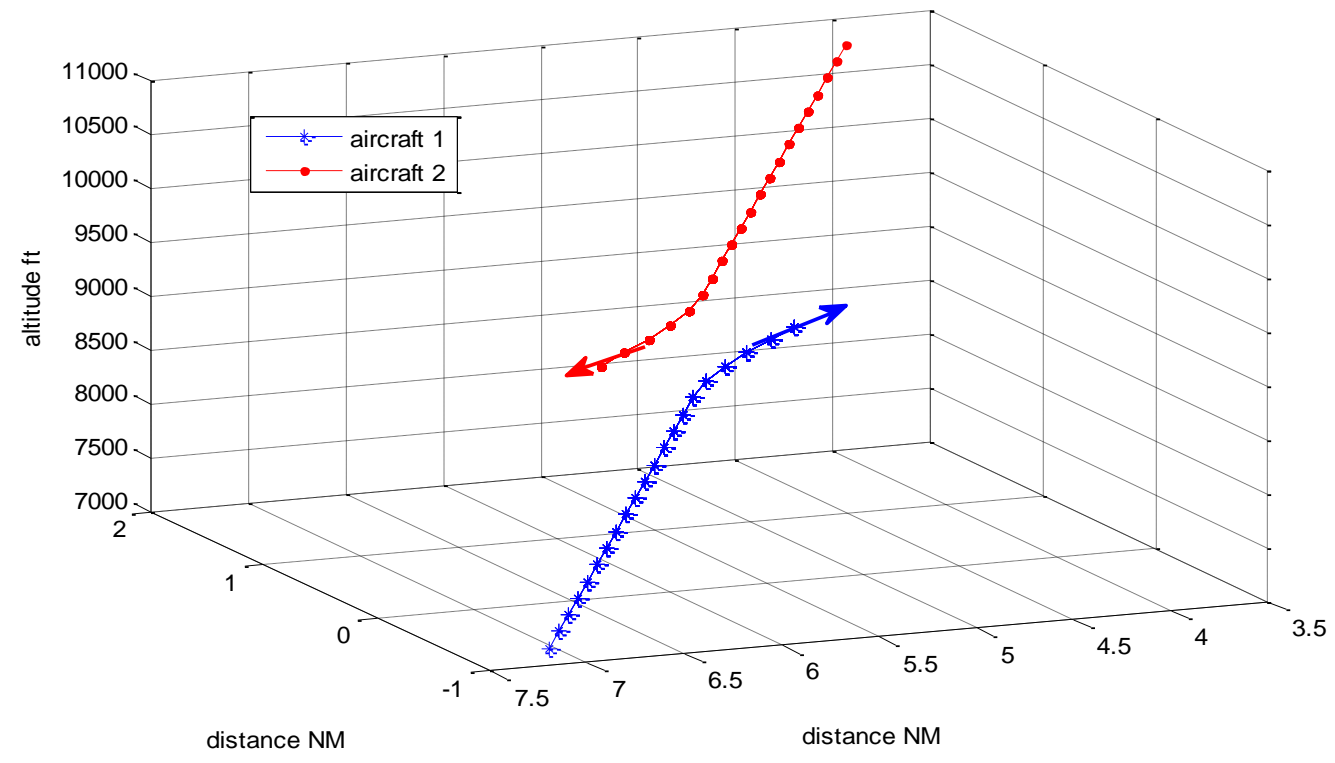

Figure 7. Aircraft Face to Face

Aircraft 1 was cleared to climb to FL110 and aircraft 2 was cleared to descend to FL070, both aircraft are flying face to face. As aircraft 1 and 2 approach the cleared level FL090 at a high vertical rate (and therefore threatening to bust the required separation) TCAS issues an RA to decrease vertical speed and both aircraft have to go right following rule 1, The aircraft 2 continues descending to FL070 and aircraft 2 continues climbing to FL110.

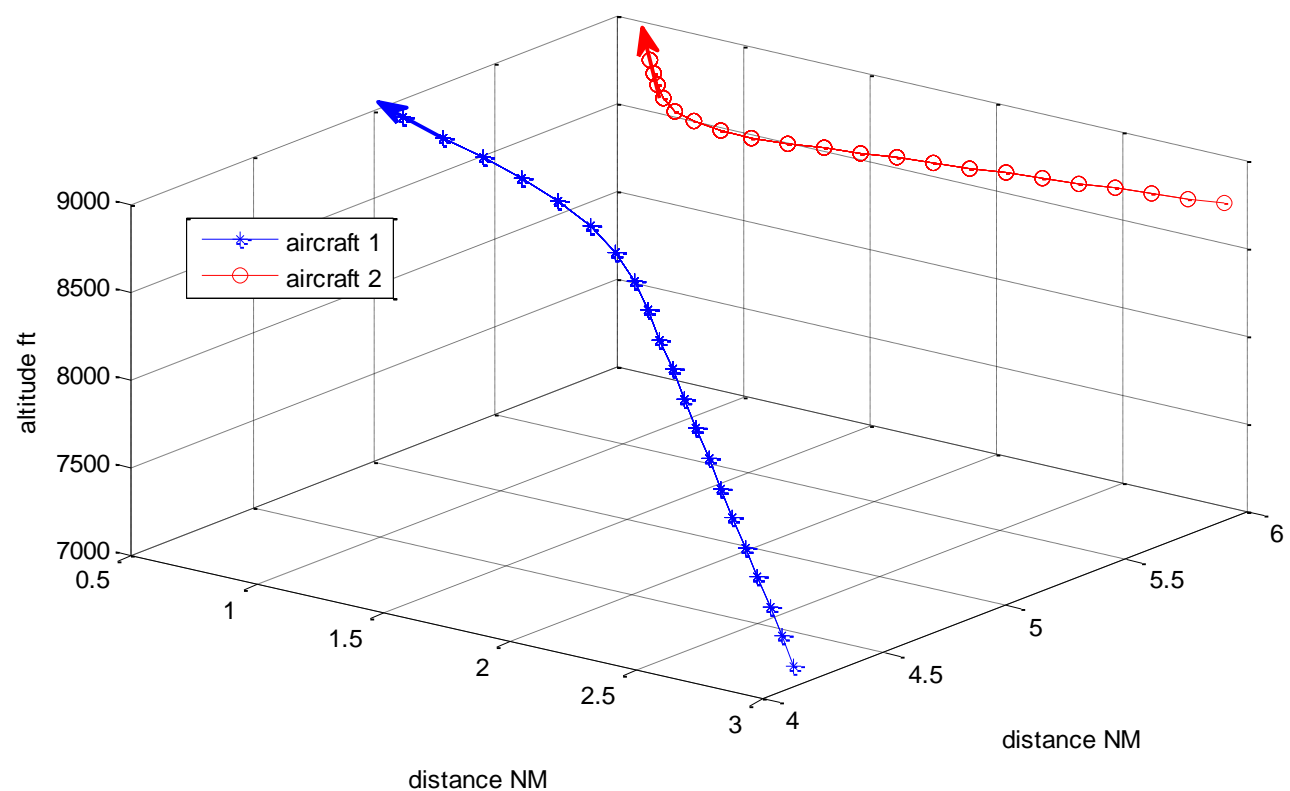

Figure 8. Vertical then Horizontal Resolution

This encounter can involve three aircraft or more. Aircraft 1 was climbing to FL090 while aircraft 2 is maintaining altitude 8800 feet. If we have an aircraft at FL100, the only way to ovoid collision is the horizontal resolution, so TCAS guides the pilot of AC1 to the left with 
adjust vertical speed and aircraft 2 to the right. Figure 8 shows how TCAS can provide guidance between two intruders handling multiple simultaneous RA's.

\section{Conclusion}

This TCAS vision has different performance capabilities and relies upon different surveillance systems from traditional TCAS aircraft. The main objective is to insure Selfseparation maneuvers which may require heading and speed changes.

Since future airspace may utilize reduced separation standards to increase efficiency. This procedure-specific functionality would allow alerting pilots and prevent interference from frequent unnecessary alerts

This service does not require infrastructure on the ground, just satellite constellation and transponders, The purpose of this work is to provide visual information using ADS-B satellite data link in cockpit. This allows pilots to make verification of the safe operation and the right maneuver and help them to operate safely with less stress especially in CTA (Control Terminal Area). In addition, this paper seeks to detect any anomalies in the safe operation of TCAS and to provide any information that will help their correction.

This paper suggests that this potential effectiveness of monitoring project be recommended by the ICAO for removing unnecessary TCAS resolution advisories.

\section{References}

[1] Annex 10 to the convention on international civil aviation, "Surveillance and Collision Avoidance systems", Aeronautical telecommunications, fourth edition, vol. 4, (2007), pp. 1-23.

[2] M. C. Stevens, "Secondary Surveillance Radar", Artech House Boston and London, (1988), pp. 10 - 50.

[3] A. M. G. Ivan, L. Mauro, G. Gaspare, V. B. T. Juan and L. R. D. Elías, "Efficient location strategy for airport surveillance using Mode-S multilateration systems", International Journal of Microwave and Wireless Technologies, vol. 4, no. 2, (2012), pp. 209-216.

[4] X. Yajun, "TCAS/ ADS-B Integrated Surveillance and Collision Avoidance System", 2nd International Conference on Computer Science and Electronics Engineering (ICCSEE), (2013); China.

[5] L. Ian and J. William, "Modeling ADS-B out System Latency”, 31st Digital Avionics Systems Conference, Atlantic City, (2012).

[6] M. J. Kochenderfer, J. E. Holland and J. P. Chryssanthacopoulos, Next-Generation Airborne Collision Avoidance System, LINCOLN Laboratory Journal, vol. 19, no. 1, (2012).

[7] Eurocontrol, “ACAS II Guide Airborne Collision Avoidance System II”, (Incorporating version 7.1), (2012), pp. $3-65$.

[8] P. Nicolas, G. Gaspare and P. Emilio, "Coherent sources separation based on sparsity: an application to SSR signals", International Journal of Microwave and Wireless Technologies, vol. 1, no. 3, (2009), pp. 185-191.

[9] C. Denis, "Navigation Générale JAR-FCL ATPL”, Institut d'aéronautique Jean Mermoz, Version 3, (2003), pp. $73-92$.

[10] E .L. Chin and W. Yi -Yu, "TCAS Solution for Low Altitude Flights", International Communications Navigation and Surveillance (ICNS) Conference, (2010); Tainan.

[11] G. Jesus, A. B. Juan, S. Andres and M. Gonzalo, "Opportunity trajectory reconstruction techniques for evaluation of ATC systems", International Journal of Microwave and Wireless Technologies, vol. 1, no. 3, (2009), pp. 231-238.

[12] E. R. Charles and R. E. Tomas, "TCAS Surveillance Algorithm Modification for Reduced Channel Utilization", 30th Digital Avionics Systems Conference, (2011) ; Massachusetts, USA. 


\section{Authors}

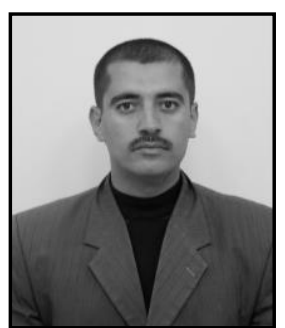

Abdelali Achachi was born in 1979. Received his Air Traffic Control licence from Higher school of military air defense (Algeria) in 2002 and worked as an ATC until 2014 with ENNA company. He received his Engineering Diploma in electronic engineering in 2004, a master degree in communication from the University of Banta (Algeria) in 2010. Currently, he is air navigation security manager and Ph.D student studying at the same university. His current research interests are: LEO satellites constellation communication system for air traffic control.

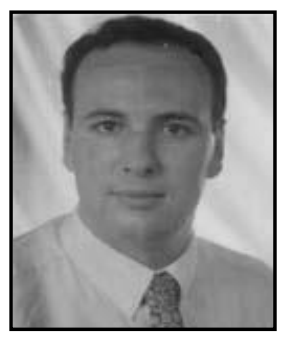

Djamel Benatia was born in Constantine (Algeria) in 1967. He received his Engineering Diploma in electronic engineering in 1990, a Master in communication in 1994 and the Ph.D in microwaves in 1999 from the Electronic Institute, University of Constantine. In 1994 he joined the Electronic Department, University of Banta (Algeria). In 2000 he received the Associate Professor degree. In 2005 he received the Professor degree.

From 2003 to 2013 he has occupied the post of President of Scientist Comity of the Electronic Department of Batna University. His research interests are: the propagation of acoustic microwaves in piezoelectric material and the transmission by satellite. 